\title{
Gravitation, Holographic Principle, and Extra Dimensions
}

\author{
Roberto Caimmi* \\ Physics and Astronomy Department, Padua University, Padova, Italy \\ Email: roberto.caimmi@unipd.it
}

Received 19 November 2015; accepted 26 February 2016; published 29 February 2016

Copyright (C) 2016 by author and Scientific Research Publishing Inc.

This work is licensed under the Creative Commons Attribution International License (CC BY). http://creativecommons.org/licenses/by/4.0/

(c) (i) Open Access

\begin{abstract}
Within the context of Newton's theory of gravitation, restricted to point-like test particles and central bodies, stable circular orbits in ordinary space are related to stable circular paths on a massless, unmovable, undeformable vortex-like surface, under the action of a tidal gravitational field along the symmetry axis. An interpretation is made in the light of a holographic principle, in the sense that motions in ordinary space are connected with motions on a selected surface and vice versa. Then ordinary space is conceived as a 3-hypersurface bounding a $n$-hypervolume where gravitation takes origin, within a $n$-hyperspace. The extension of the holographic principle to extra dimensions implies the existence of a minimum distance where test particles may still be considered as distinct from the central body. Below that threshold, it is inferred test particles lose theirs individuality and "glue" to the central body via unification of the four known interactions and, in addition, 1) particles can no longer be conceived as point-like but e.g., strings or membranes, and 2) quantum effects are dominant and matter turns back to a pre-big bang state. A more detailed formulation including noncircular motions within the context of general relativity, together with further knowledge on neutron stars, quark stars and black holes, would provide further insight on the formulation of quantum gravity.
\end{abstract}

\section{Keywords}

Newtionian Theory of Gravitation, Holographic Principle, Extra Dimensions

\section{Introduction}

According to current QCDM cosmologies, the universe as a whole is increasingly expanding e.g., [1] which cannot be true for subsystems. For instance, massive (exceeding about 20 solar masses) stars at the end of evolution are no longer pressure-balanced and collapse into a black hole e.g., [2] where matter may safely be con-

*The author is in retirement due to age limits. 
ceived as turned into a pre-big bang state, implying unification of the four known interactions as predicted by supersymmetric theories e.g., [3]. In the light of general relativity, the final configuration is point-like but the occurrence of quantum effects could yield strings or membranes, leaving the gravitational field outside the instability region (where test particles cannot remain in stable equilibrium) spherical-symmetric. Deeper investigation on neutron stars and quark stars (if their existence is proved), which are celestial bodies most similar to black holes, could provide further insight on the formulation of quantum gravity.

A simplified description can be made within the framework of Newton's theory of gravitation, where spherical-symmetric matter distributions can be conceived as point-like outside their boundaries. The concept of space curvature may be introduced considering a vortex-like surface under the action of a tidal gravitational field along the symmetry axis, where the distance of a surface point from the symmetry axis relates to the distance of a test particle from a central body. The current paper aims to answer two specific questions restricted, for simplicity, to circular motions.

First, could stable circular orbits (radius, $R$ ) described by a test particle (mass, $m$ ) around a central body (mass, $M$ ) be related to stable circular paths described by a test particle (mass, $m$ ) on a massless, unmovable, undeformable vortex-like surface, subjected to a constant gravitational force, $-G M m / R^{2}$, directed downwards along the symmetry axis? If yes, a principle of corresponding states could be expressed as a holographic principle in the sense that motions in ordinary space are connected with motions on a selected surface and vice versa.

Second, could the above mentioned holographic principle be extended to ordinary space, conceived as a 3-hypersurface, where test particles are subjected to a constant gravitational force from extra dimensions? If yes, could quantum effects and unification of the four known interactions be interpreted in this context?

The first and second questions raised are dealt with in Sections 2 and 3, respectively. The discussion is drawn in Section 4. The conclusion is shown in Section 5. A special vortex-like surface and an analogy for the invariance of light velocity are presented in the Appendix A1 and Appendix A2, respectively.

\section{A Holographic Principle}

Let a test particle of mass, $m$, move along a stable circular orbit of radius, $R$, around a central body of mass, $M$. Let both the test particle and the central body be conceived as point-like. The balance of gravitational and centrifugal force at a generic point of the orbit, after little algebra reads:

$$
V^{2}=\frac{G M}{R}
$$

where $G$ is the constant of gravitation and $V$ the circular velocity.

Let a vortex-like, massless, unmovable, undeformable surface (in short, surface) be subjected to a tidal gravitational field along the symmetry axis, implying constant attraction directed downwards, as depicted in Figure 1.

Let the surface be defined as $z=f(w), w^{2}=x^{2}+y^{2}$, with the following boundary conditions:

$$
\lim _{w \rightarrow \mp \infty} \frac{\mathrm{d} f}{\mathrm{~d} w}=0^{ \pm} ; \quad \lim _{w \rightarrow 0^{\mp}} \frac{\mathrm{d} f}{\mathrm{~d} w}= \pm \infty ;
$$

where the vertical axis is directed downwards. Let $\mathrm{P} \equiv(w, z)$ be a generic point on the surface; $w_{0}, z_{0}$, the intercepts of a tangent to the surface, lying on a meridional plane and crossing $\mathrm{P}$, and $\theta$ the angle defined by the tangent and the axis, $w$, see Figure 1.

The equation of the tangent is:

$$
\begin{gathered}
w_{0} z+z_{0} w=w_{0} z_{0} ; \\
\tan \theta=-\frac{z_{0}}{w_{0}}=-\frac{z_{0}-z}{w} ; \quad-w \tan \theta>0 ;
\end{gathered}
$$

where $\theta \rightarrow 0^{ \pm}$implies $w \rightarrow \mp \infty, z \rightarrow 0^{+}$, and $\theta \rightarrow(\pi / 2)^{\mp}$ implies $w \rightarrow 0^{\mp}, z \rightarrow+\infty$. The evaluation of $\theta$ needs the knowledge of the surface equation.

With regard to a test particle on a selected point, $\mathrm{P}$, of the surface, the gravitational force, $F_{g}$, can be splitted into a normal component, $F_{g} \cos \theta$, balanced by the reaction due to surface unmovability and undeformability, and a tangential component, $F_{g} \sin \theta$, directed downwards i.e. towards increasing $z$, see Figure 1 . Similarly, 


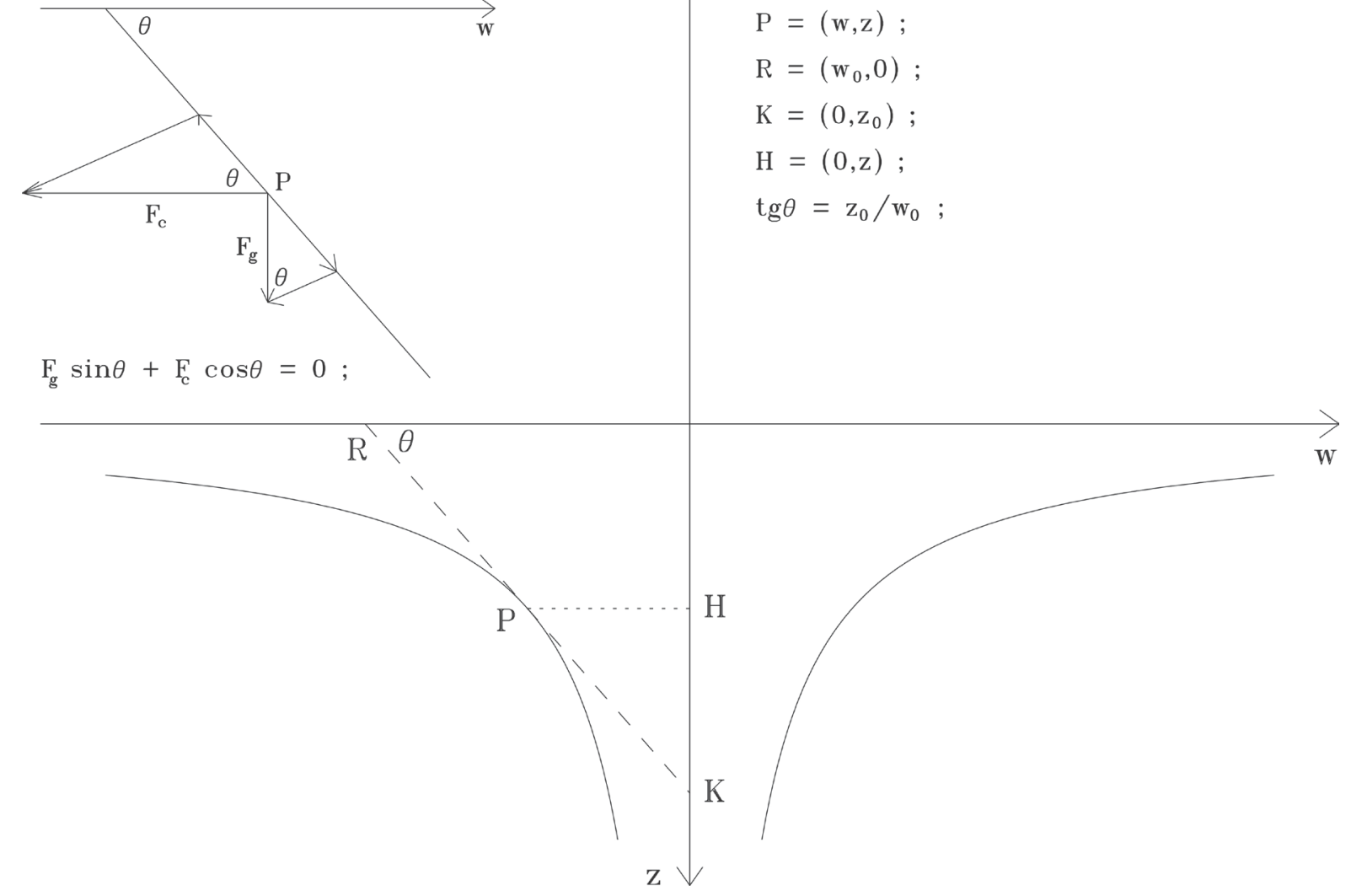

Figure 1. A vortex-like, massless, unmovable, undeformable surface subjected to a tidal gravitational field along the symmetry axis, implying constant attraction directed downwards. The axis, $w, w^{2}=x^{2}+y^{2}$, lies on the horizontal plane. The axis, $z$, coincides with the symmetry axis i.e. the vertical direction, related to free fall. The angle, $\theta$, is formed by the axis, $w$, and the tangent to the surface, lying on a meridional plane and crossing a selected point, P. The tangential component of the gravitational attraction, $F_{g}$, and the centrifugal repulsion, $F_{c}$, related to a test particle on P moving along a stable circular path, $(w, z)$, are $F_{g} \sin \theta$ and $F_{c} \cos \theta$, respecttively. The angle, $\theta$, can be inferred from the equation of the surface, $z=f(w)$, where $\lim _{w \rightarrow \mp \infty} \mathrm{d} f / \mathrm{d} w=0^{ \pm}$and $\lim _{w \rightarrow 0^{\mp}} \mathrm{d} f / \mathrm{d} w= \pm \infty$. The slope of the tangent is positive in the third quadrant and negative in the fourth quadrant, as the $z$ axis is oriented downwards. The curves plotted are branches of equilateral hyperbola, $w z=\mp 10$. The coordinates of interest and the angle, $\theta$, are specified on the upper right. The force balance on a test particle on the point, $\mathrm{P}$, is shown on the upper left.

the centrifugal force, $F_{c}$, can be splitted into a normal component, $F_{c} \sin \theta$, balanced by the reaction due to surface unmovability and undeformability, and a tangential component, $F_{c} \cos \theta$, directed upwards i.e. towards increasing $|w|$, see Figure 1.

Motion along a stable circular path, $|w|=$ const, $z=$ const, implies $F_{g} \sin \theta+F_{c} \cos \theta=0$. The choice, $F_{g}=G M m / R^{2}$, translates into:

$$
v^{2}=-\frac{G M}{R} \frac{w}{R} \tan \theta=\frac{G M}{R} \frac{z_{0}-z}{R} ;
$$

where $v$ is the circular velocity.

The combination of Equations (1) and (5) yields:

$$
\frac{v^{2}}{V^{2}}=-\frac{w}{R} \tan \theta=\frac{z_{0}-z}{R} ;
$$

and the substitution of Equation (4) into Equation (6) produces: 


$$
w=\frac{v^{2}}{V^{2}} \frac{R}{z_{0}} w_{0}
$$

where the intercepts of the equation of the tangent, $w_{0}$ and $z_{0}$, see Figure 1 , can be determined using the surface equation, $z=f(w)$. In the special case where the meridional section of the surface shows branches of equilateral hyperbola, one finds $w_{0} / z_{0}=w / z$ and Equation (7) reduces to:

$$
\frac{z}{R}=\frac{v^{2}}{V^{2}}
$$

for a formal demonstration, an interested reader is addressed to Appendix A1.

The above results may be summarized into a single statement.

Holographic principle. Given a central body of mass, $M$, and a vortex-like, massless, unmovable, undeformable surface subjected to a tidal gravitational field along the symmetry axis, implying constant attraction directed downwards, any test particle moving on a stable circular orbit of radius, $R$, and velocity, $V$, has a counterpart on the vortex-like surface, moving along a stable circular path of radius, $|w|$, and velocity, $v$, around the symmetry axis, where $w=(v / V)^{2}\left(R / z_{0}\right) w_{0}$.

In general, the above statement should be conceived as a principle of corresponding states: the term "holographic" has to be intended in the sense that motions in ordinary space are connected with motions on a selected surface and vice versa.

The above considerations could, in principle, be extended to general relativity but, for simplicity, are restricted to classical mechanics, which implies velocities up to infinity. If light velocity is assumed as an empirical upper limit, $v \leq c$, stable orbits cannot occur below a critical radius, $R_{c}$, expressed via Equation (1) as:

$$
R_{c}=\frac{G M}{c^{2}}
$$

according to Newton's theory of gravitation. Its counterpart in general relativity, using Schwarzschild's metric, reads e.g., [4]:

$$
R_{c}=\frac{3 G M}{c^{2}}
$$

which is three times larger.

Similarly, stable circular paths on a vortex-like surface, centered on the symmetry axis, cannot occur below a critical radius, $\left|w_{c}\right|$, expressed via Equations (7) and (9) as:

$$
w_{c}=R_{c} \frac{w_{0}}{z_{0}}=\frac{G M}{c^{2}} \frac{w_{0}}{z_{0}}
$$

where $v=V=c$.

\section{Gravitation and Extra Dimensions}

Let ordinary space be conceived as a 3-hypersurface bounding a $n$-hypervolume, totally $(n=4)$ or partially $(n>4)$. Let the source of the gravitational field lie within the hypervolume and let the hypersurface be curved in presence of matter via gravitational interaction. To this respect, the following analogy can help.

With regard to a planet surrounded by a liquid shell, a global ocean say, the source of the gravitational field within the hypervolume is represented by the whole planet and matter within the hypersurface by the water surface, respectively. In absence of matter (empty ordinary space), the water surface is totally flat. In presence of negligible amount of matter, the water surface can be considered flat to a good extent. In presence of considerable amount of matter, the water surface is curved by gravitational interaction. A test particle on the curved surface is subjected to the tangential component of the gravitational force, as the normal component is balanced by the surface tension. In principle, the curvature angle with respect to the horizontal plane ranges from $\theta=0$ (vortex top) to $\theta=\mp \pi / 2$ (vortex bottom).

Keeping in mind the above mentioned analogy, let the whole range of curvature be represented as an equilateral hyperbolic funnell i.e. a revolution figure related to the rotation of the bottom branch of an equilateral hyperbola around the axis, $z$, as depicted in Figure 1, where the axis, $w, w^{2}=x^{2}+y^{2}$, lies on the horizontal 
plane and the axis, $z$, is directed along the free-fall direction within the hyperspace. Accordingly, the equation of the funnell, via Equation (18) in Appendix A1, reads:

$$
\left(x^{2}+y^{2}\right) z^{2}=k^{2} ; \quad z>0 ;
$$

due to the symmetry with respect to the rotation axis, $z$.

Let a test particle of mass, $m$, move on a stable circular orbit of radius, $R$, around a central body of mass, $M$, both conceived as point-like for simplicity. Let $F_{g}, F_{G} ; F_{c}, F_{C}$; denote gravitational and centrifugal forces acting on the test particle within the hypersurface (ordinary space) and hypervolume (hyperspace), respectively, which implies the following relations:

$$
\begin{aligned}
& F_{G} \sin \theta+F_{C} \cos \theta=0 ; \\
& F_{g}=F_{G} \sin \theta=-\frac{G M m}{R^{2}} ; \\
& F_{c}=F_{C} \cos \theta=\frac{m V^{2}}{R} ;
\end{aligned}
$$

where $G$ is the constant of gravitation and $V$ the orbital velocity. The combination of Equations (13)-(15) yields Equation (1) and, in particular, Equation (9) assuming light velocity as an empirical upper limit.

The gravitational force within the hypervolume via Equation (14) reads:

$$
F_{G}=-\frac{G M m}{R^{2} \sin \theta}
$$

where $R^{2} \sin \theta=$ const according to the assumption of hypersurface where test particles are subjected to a constant gravitational force, $F_{G}$, from extra dimensions along the symmetry axis. Then $\theta \rightarrow 0$ implies $R \rightarrow+\infty$ and, in addition, $R^{2} \sin \theta=R_{0}^{2}$, where $R_{0}$ relates to $\theta=\pi / 2$. In general, the following relation holds:

$$
\theta=\arcsin \left(\frac{R_{0}}{R}\right)^{2}
$$

in terms of the orbit radius, $R$. On the other hand, $R_{0} \rightarrow 0$ can be inferred from general relativity e.g., [5], and a nonzero $R_{0}$ can be interpreted as due to quantum effects, which act to "close" the bottom of the funnell.

Let a finite matter distribution, spherical-symmetric for simplicity, be defined by a total mass, $M$, within a finite radius, $R$. Accordingly, curvature effects are directly proportional to the angle, $\theta$, between the horizontal axis and the tangent on a selected point, with regard to a meridional section of the funnell, as depicted in Figure 2.

Curvature effects are larger on the surface, which can be related to the angle, $\theta=\theta_{\text {inf }}$, involving the inflexion point of related meridional section. Within the matter distribution, curvature effects are reduced due to Newton's theorem e.g., [6] and removed at the centre, where no gravitational attraction takes place as (in the current interpretation) the gravitational force, $F_{G}$, has null tangential component.

The velocity of a test particle in a stable circular orbit, on the surface of the matter distribution, is increasing for decreasing $R$ until $V=c$ for $R=R_{c}$. Lower radii, $R<R_{c}$, would imply a vortex-like distorsion but the bottom of the funnel is expected to be "closed" by quantum effects behind a threshold, $R=R_{\min } \geq R_{0}$, related to $\theta=\theta_{\max } \leq \pi / 2$. For $R<R_{\min }, \theta<\theta_{\max }$, up to $R=0, \theta=0$, where no gravitational attraction takes place, as expected. Accordingly, $\theta_{c} \leq \theta_{\max } \leq \pi / 2$, which implies $R_{c} \geq R_{\min } \geq R_{0}$, via Equation (17). If, in particular, $R_{c}=(3 / 2) R_{g}, R_{\min }=R_{g}$, where $R_{g}=2 G M / c^{2}$, with regard to Schwarzschild black holes in general relativity e.g., [7] (Chap. XI, §97), then Equation (17) reduces to $\theta_{c}=\arcsin \left(R_{\min } / R_{c}\right)^{2}=\arcsin (4 / 9) \approx 0.1466 \pi$.

In summary, stable circular orbits of test particles around a central body can occur for $R \geq R_{c}$, implying $V \leq c$, while the motion of test particles is unstable for $R<R_{c}$ up to $R=R_{\min }$. Beyond that threshold, quantum effects are expected to be dominant and test particles are "glued" to the central body. 


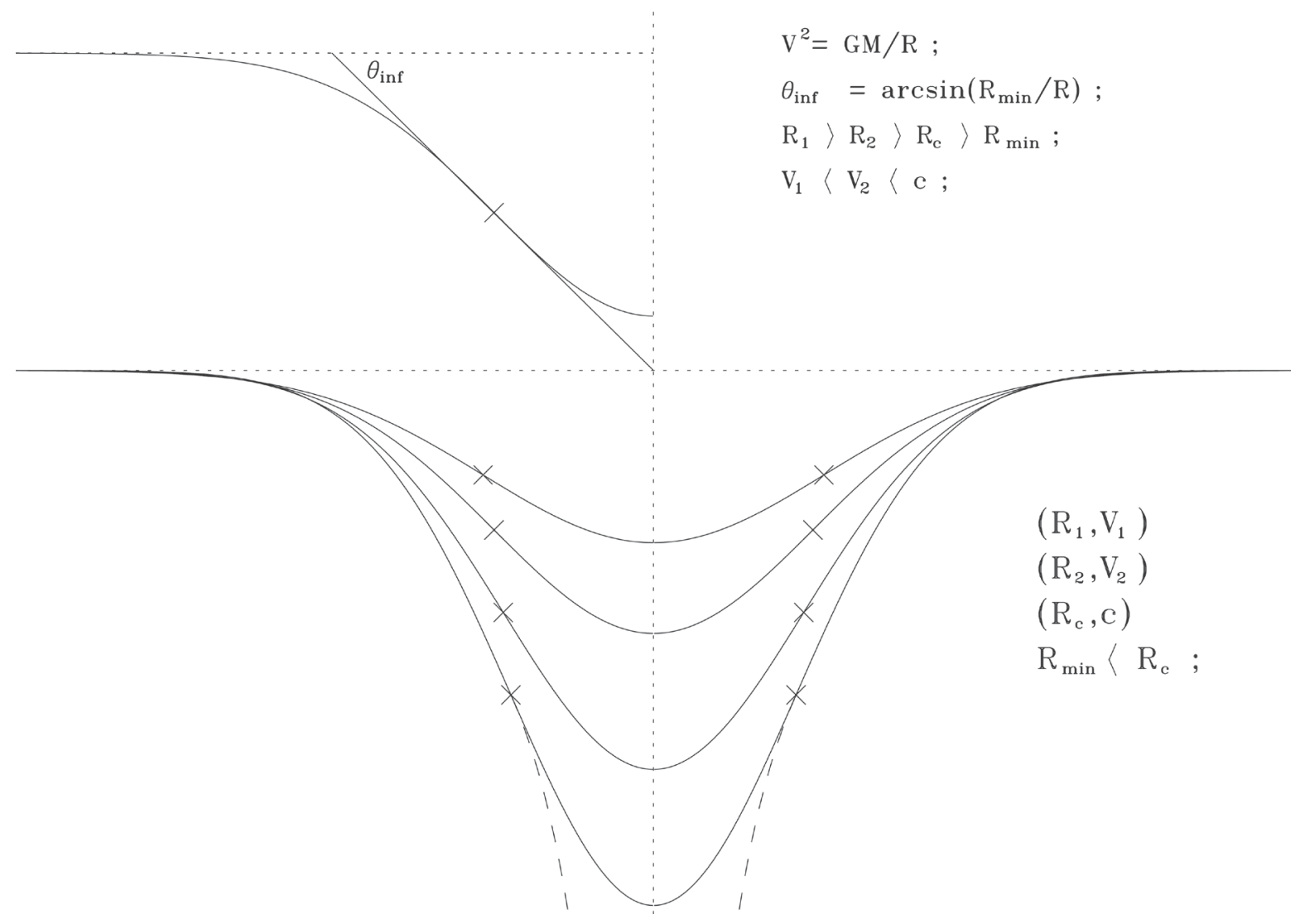

Figure 2. Qualitative representation of curvature effects induced by a finite matter distribution, spherical-symmetric for simplicity, defined by a total mass, $M$, within a finite radius, $R$. Curvature effects are larger on the inflexion point of a meridional section of the funnell. A test particle in a stable circular orbit on the surface of the matter distribution has velocity, $V$, inversely proportional to the radius, $R$, until $V=c$ for $R=R_{c}$. Lower radii, $R<R_{c}$, would imply a vortex-like distortion (dashed) in absence of quantum effects, which act to "close" the bottom of the funnell up to $R=R_{\min } \geq R_{0}$, where the angle between the tangent on the inflexion point and the horizontal axis, with regard to a meridional section (upper left), is $\theta_{\text {inf }}=\theta_{\max } \leq \pi / 2$. The meridional section of the funnel has been arbitrarily plotted as an upturned Gaussian, $z=-a \exp \left(-b w^{2}\right)$, where $(a, b)=(3.8,0.07),(5.8,0.08),(8.8,0.09),(9.8,0.10)$, from top to bottom, and the inflexion points are marked by saltires. Dashed curves are branches of equilateral hyperbola passing through the inflexion points of the bottom curve. See text for further details.

\section{Discussion}

According to the holographic principle inferred in Section 2, stable circular orbits of a test particle of mass, $m$, around a central body of mass, $M$, can be related to stable circular paths of a test particle of mass, $m$, on a vortex-like, massless, unmovable, undeformable surface, subjected to a tidal gravitational field along the symmetry axis, implying constant attraction directed downwards. In other words, the gravitational action on the ordinary space can be related to the gravitational action on an axisymmetric surface. By analogy, the gravitational action on a $n$-hyperspace can be related to the gravitational action on an axisymmetric 3-hypersurface, coinciding with the ordinary space. For reasons of simplicity, considerations have been restricted to circular motions within Newton's theory of gravitation, but it can safely be expected an extension to generic motions within the theory of general relativity.

If gravitational attraction takes origin from a $n$-hypervolume, the origin of (ordinary) space curvature is no longer due to matter in itself, but to gravitation from extra dimensions. Then matter appears to be entirely passive, in the sense that it can no longer be thought of as source of gravitational field. In this view, extra dimensions extend as well as ordinary ones, contrary to assumptions of superstring theories, where extra dimensions 
are highly compressed e.g., [3].

The main consequence of the above mentioned analogy is the occurrence of quantum effects, in the sense that a test particle can approach a central body (both considered as point-like) up to a threshold, $R=R_{\min }$, preserving its intrinsic properties. A physical interpretation could be the following. First, for sufficiently short distances, masses can no longer be conceived as point-like, but perhaps as strings or membranes, similarly to charged particles e.g., protons and electrons, where the electric charge cannot be conceived as point-like. Second, for sufficiently large densities, the four known interactions are expected to be unified into a superinteraction, according to supersymmetric theories e.g., [3], where particles could be "glued" similarly to quarks into adrons. In other words, the occurrence of a superinteraction implies matter returns to a pre-big bang state.

\section{Conclusions}

Within the context of Newton's theory of gravitation, for an assigned (point-like) test particle and (point-like) central body, stable circular orbits in ordinary space have been related to stable circular paths on a vortex-like, massless, unmovable, undeformable surface, subjected to a tidal gravitational field along the symmetry axis, implying constant attraction directed downwards, and a holographic principle has been proposed. If ordinary space is conceived as a 3-hypersurface bounding a $n$-hypervolume where gravitational interaction takes origin, the extension of the above mentioned holographic principle has implied a minimum distance behind which test particles lose theirs individuality (intended as collection of intrinsic properties) and "glue" to the central body via unification of the four known interactions, behind which 1) particles can no longer be conceived as point-like but perhaps as strings or membranes; and 2) quantum effects are dominant and matter returns to a pre-big bang state.

The generalization of the procedure to noncircular motions within the context of general relativity, together with further knowledge about neutron stars, quark stars, black holes, e.g., [8] could provide further insight on the formulation of quantum gravity.

\section{Acknowledgements}

Thanks are due to the Editor and the referee for their comments.

\section{References}

[1] Percival, W.J. (2005) Astronomy and Astrophysics, 443, 819-830. http://dx.doi.org/10.1051/0004-6361:20053637

[2] Morscher, M., Pattabiraman, B., Rodriguez, C., Rasio, F.A. and Umbreit, S. (2015) The Astrophysical Journal, 800, Article ID: 9 (21 pp).

[3] Greene, B. (1999) The Elegant Universe. W. W. Norton \& Company, New York.

[4] Zeldovich, Ya.B. and Novikov, I.D. (1971) Relativistic Astrophysics. University of Chicago Press, Chicago.

[5] Misner, C.W., Thorn, K.S. and Weeler, J.A. (1972) Gravitation. W. H. Freeman \& Company, New York.

[6] MacMillan, W.D. (1930) The Theory of the Potential. Dover Publications, Inc., New York.

[7] Landau, L. and Lifschitz, E. (1966) Théorie du Champs. Mir, Moscow.

[8] Lugones, G. and Arbañil. J.D.V. (2015) Astronomische Nachrichten, 336, 876-879. http://dx.doi.org/10.1002/asna.201512241

[9] Caimmi, R. (2008) New Astronomy, 13, 261-284. http://dx.doi.org/10.1016/j.newast.2007.10.005

[10] Messiah, A. (1966) Mécanique Quantique. Dunod, Paris. 


\section{Appendix}

\section{A1. A Guidance Example}

As a guidance example, let the meridional section of the vortex-like surface coincide with branches of equilateral hyperbola, as:

$$
w Z=\mp k
$$

where the right-hand side is positive or negative according if the hyperbola branch lies on the fourth or third quadrant, respectively, see Figure 1. Let $P \equiv\left(w_{P}, z_{P}\right)$ be a generic point on the hyperbola.

The intersection of the hyperbola with a generic straight line, expressed as:

$$
z=a w+b ;
$$

can be inferred via substitution of Equation (19) into Equation (18), as solution of the related second-degree equation in $w$. The result is:

$$
w_{P}=\frac{-b \mp \sqrt{b^{2} \mp 4 a k}}{2 a} ;
$$

where the double signs are uncorrelated.

The straight line is tangent to an hyperbola branch provided the discriminant is null, as:

$$
b^{2} \mp 4 a k=0 ; \quad \mp a k<0 ; \quad \pm a k>0 ;
$$

accordingly, the coordinates of the tangential point are:

$$
\begin{gathered}
w_{P}=-\frac{b}{2 a}=-\frac{\sqrt{ \pm a k}}{a} ; \\
z_{P}=\mp \frac{k}{w_{P}}=\frac{ \pm a k}{\sqrt{ \pm a k}}=\sqrt{ \pm a k} ;
\end{gathered}
$$

from which the slope and the intercepts of the tangent straight line are inferred via Equations (18) and (19), as:

$$
\begin{gathered}
a=\frac{z_{P}^{2}}{ \pm k}=-\frac{z_{P}}{w_{P}} ; \\
w_{0}=-\frac{b}{a}=2 w_{P} ; \\
z_{0}=b=-2 a w_{P}=2 z_{P} ;
\end{gathered}
$$

in terms of the coordinates of the tangential point, $P$. Finally, the substitution of Equations (25) and (26) into (4) yields:

$$
\tan \theta=-\frac{z_{P}}{w_{P}} ;
$$

accordingly, $\theta$ is positive or negative if related to an hyperbola branch lying on the third or the fourth quadrant, respectively, see Figure 1.

The substitution of Equations (25) and (26) into (7) after little algebra produces:

$$
z_{P}=\frac{v^{2}}{V^{2}} R ;
$$

which is equivalent to Equation (8) and, in particular, $v=V$ implies $z_{P}=R ; v=V=C$ implies $Z_{P}=R_{c}=G M / c^{2}$.

\section{A2. An Analogy for Light Velocity Invariance}

Let bodies (particles, in particular) be conceived as vessels moving on a global ocean. Let light be conceived as a circular wave on the ocean which can be induced via e.g., vessel oscillations along the vertical direction. Then 
waves propagate on the ocean surface, regardless of vessel motion. The concept of "light velocity in vacuum" appears to be meaningless e.g., [9] in that, according to current cosmologies, space is permeated by a sort of dark energy e.g., [1] and, on the other hand, quantum vacuum is also permeated by (minimum) energy e.g., [10].

Conceptually, wave velocity can be determined in the following way. Let vessels move between equally spaced series of buoies, similarly to electric trains between high-voltage tralisses. Let $\Delta s$ be the distance between adjacent buoies (along motion direction), and let $\Delta t$ be the time of wave propagation between adjacent buoies (along motion direction) which, in principle, can be determined from vessels. Accordingly, wave velocity can be inferred as $v_{w}=\Delta s / \Delta t$, regardless of vessel motion i.e. reference frame comoving with a vessel.

In addition, wave velocity cannot be exceeded by vessel velocity, $v<v_{w}$. The limit, $v \rightarrow v_{w}$, would imply vessels be turned into water, where waves can propagate.

Finally, the reference frame comoving with the ocean i.e. at rest with respect to the ocean can be conceived as "privileged".

As mentioned in Section 3, ordinary space can be related to ocean surface and extra dimensions to ocean volume. 